Having read this article, the reader will:

- Appreciate the contribution of Pierre Fauchard to the development of the modern practice of maxillofacial prosthodontics.

- Appreciate the historical difficulties experienced by patients suffering from defects of the hard and soft palate.

- Appreciate the historical challenges faced by dental practitioners when rehabilitating patients with defects of the hard and soft palate.

\title{
Pierre Fauchard and his rôle in the development of obturators
}

\author{
C. D. Lynch, ${ }^{1}$ C. T. MacGillycuddy ${ }^{2}$ and V. R. O'Sullivan ${ }^{3}$
}

\begin{abstract}
The design and fabrication of oral appliances to replace parts of the palate missing due to congenital defects or lost through tumours, infection or trauma has been a considerable challenge for clinicians throughout the history of dentistry. Significant advances were made during the eighteenth century towards resolving the problem of constructing satisfactory obturators by the first 'surgeon-dentist', Pierre Fauchard. This paper reviews his innovative designs.
\end{abstract}

\section{INTRODUCTION}

The presence of a palatal defect can cause functional, aesthetic, and social distress for patients. ${ }^{1}$ The provision of a suitable prosthesis that obturates the defect presents clinical and technical challenges to the clinician, ${ }^{2}$ but in recent times useful progress in the provision of satisfactory obturator appliances has been made. The reasons for this include the development of techniques for making impressions of defects; the introduction of new materials from which appliances can be constructed; the use of reliable dental implants; and the establishment of closer cooperation between the surgeon and the maxillofacial prosthodontist. ${ }^{3}$ It is important to appreciate that palates affected by defects provide limited tissue support for any prosthesis that rests on them. Local invasion by tumours or infections limits the potential to use features such as the depth of sulci, muscle function or neuromuscular control, which can improve retention. ${ }^{2}$

\footnotetext{
$1^{*}$ Registrar in Restorative Dentistry, Department of Restorative Dentistry, National University of Ireland, Cork, Ireland: ${ }^{2}$ Postgraduate Student, Department of French, National University of Ireland, Cork, Ireland; ${ }^{3}$ Professor of Anatomy, RSCl Medical University of Bahrain, Bahrain ${ }^{*}$ Correspondence to: Christopher D. Lynch Email:c.lynch@ucc.ie
}

\section{Refereed Paper}

Accepted 10 August 2005

doi: 10.1038/sj.bdj.4812926

๑ British Dental Journal 2005; 199: 603-605
Nowadays, palatal defects occur as a result of surgical resection of tumours, trauma or congenital defects. ${ }^{3}$ In the eighteenth century such defects were mainly the result of syphilis or trauma. Children born with congenital clefts did not usually survive, owing to a failure to nurse. ${ }^{4}$ At that time, the practice of providing obturators was novel and the available devices were quite primitive. There were no detailed descriptions available of how they were to be constructed. The appliances were made from metals, ceramics or leather. The concept of impression making (using wax) was not described until later in the eighteenth century. ${ }^{4}$ However, considerable advances were being made in the practice of dentistry by Pierre Fauchard (1678-1761). He styled himself a chirurgien-dentiste (surgeon dentist), the first practitioner to use this title, and was responsible for developing some new and ingenious designs for obturators which greatly improved the outlook for those patients unfortunate enough to require them. ${ }^{5}$

\section{Pierre Fauchard}

Pierre Fauchard (Fig. 1) was born in Brittany, France, in $1678 .{ }^{5}$ He was originally apprenticed as a surgeon in the French Navy, following which he began working as a dentist in Western France. He read widely and studied crafts such as watchmaking and jewellery, from which he adapted techniques and instruments that

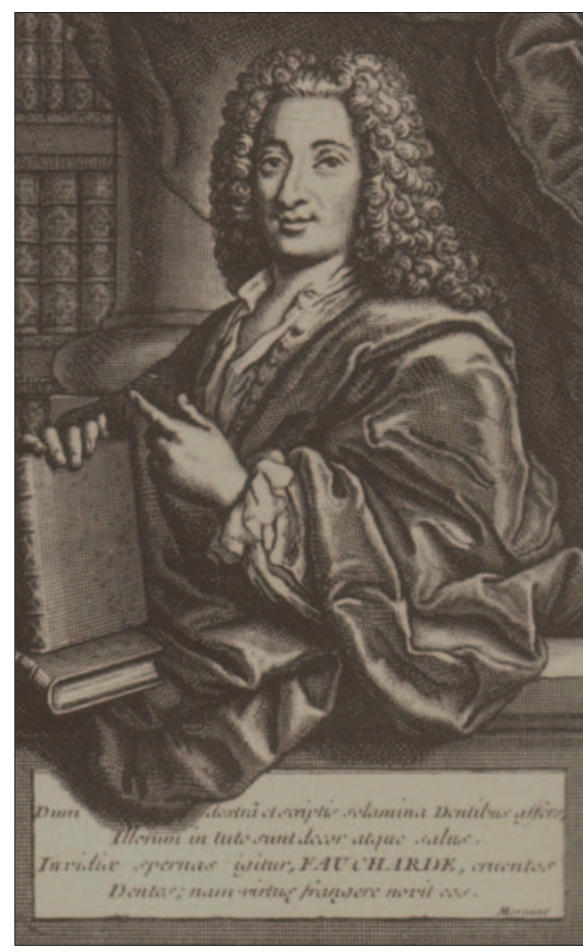

Fig. 1 Portrait of Pierre Fauchard

he felt useful to his practice. ${ }^{6}$ Fauchard's unprecedented competency and conscientiousness earned him an outstanding reputation, attracting discerning patients who journeyed great distances to see him. ${ }^{7}$ In 1719 he established a practice at the University Circle in Paris. ${ }^{5}$ Eighteenth century Paris was regarded as the centre of learn- 
ing and enlightenment in Europe. Quickly recognised as a dentist of unparalleled skill and acumen, Fauchard's advice and talents were regularly in demand by some of the city's most accomplished surgeons.

The practitioners of dentistry at that time could broadly be described in two categories. ${ }^{8}$ The first was the untrained 'toothpuller' who attended village fairs, and on whom the poor relied. These frequently employed the use of magic potions and spells to treat dental diseases and toothaches. Members of the middle and upper classes could afford the skills of more reputable practitioners who had some form of training, albeit in surgery rather than in dentistry. The practice of dentistry in these circles had been stifled by jealousy. ${ }^{5}$ Knowledge and techniques were regarded as valuable property by the more competent and skilled practitioners, and were rarely shared with colleagues. In a significant break with tradition, Fauchard published a textbook in 1728 entitled Le Chriurgien Dentiste ou Traité des Dent (The Surgeon Dentist or Treatise on the Teeth). ${ }^{5}$ This comprised two volumes of over 800 pages and included many illustrations. In this way Fauchard shared his considerable knowledge and wealth of observations with colleagues and students, describing his techniques with exceptional clarity and detail. Many of his suggestions were radical for practice in those days. For example, he advised the seating of patients for dental procedures when the conventional approach was to lay the patient on the floor. He demonstrated the presence of roots on deciduous teeth, something that was not readily accepted at the time. ${ }^{5}$ Fauchard's openness was unprecedented. Subsequent editions of his textbook appeared in both French and German over the following years, but the first English translation was not published until 1946.

Fauchard adopted a rigorously scientific approach to his practice. For example, the presence of worms as the causative agent in dental decay had been an accepted fallacy for centuries. Fauchard demonstrated that this was erroneous, as he studied decayed teeth with a magnifying glass and was unable to detect the presence of worms within the carious lesion. ${ }^{6}$ His dedication to precise and logical thinking, his conscientiousness in treating patients and his clinical skills led to his name becoming a byword for excellence in the practice of dentistry. The 'Pierre Fauchard Academy' was founded in his honour in the 1930s as an international dental honour society to recognise and acknowledge the more dedicated and conscientious dentists throughout the world. ${ }^{9}$

Fauchard's designs for palatal obturators Earlier workers in the sixteenth century, such as Franz Renner (c.1510-1577), Ama-

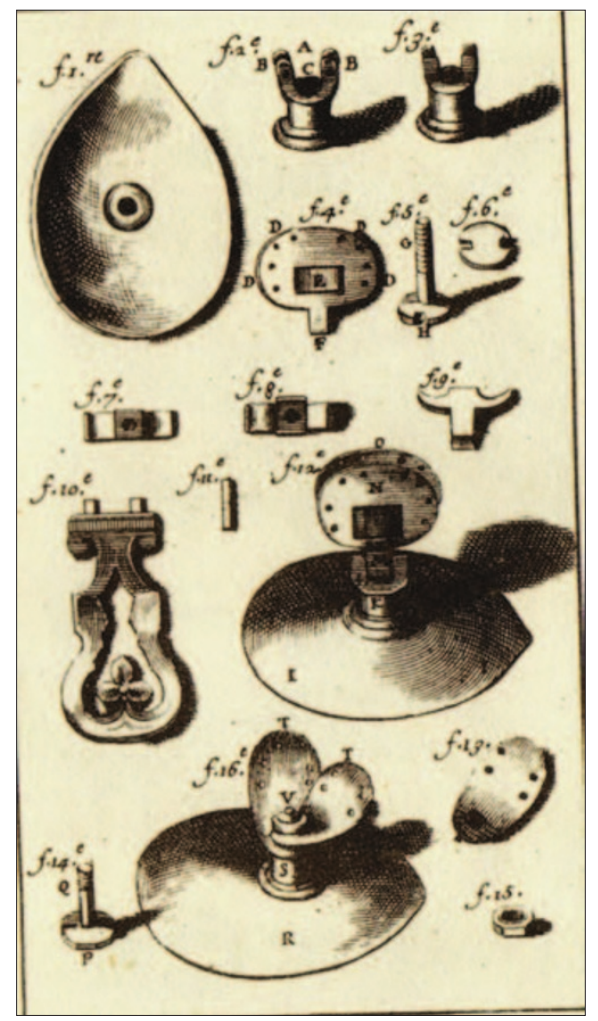

Fig. 2 Plate 38 from Le Chriurgien Dentiste. The first two designs for dentate patients are shown in the lower half of this image. The oral component of these was made from metal. The wings visible in their vertical, and subsequently lowered horizontal position, are evident in the prostheses shown in the lower half of this image.

tus Lusitanus (1511-1568), and Ambroise Paré (1510-1590) had described the use of obturators. ${ }^{4}$ Renner, who was one of the first to describe the use of obturators in modern times, used sheets of leather, ivory, gold and silver in making prostheses to obturate defects arising as a result of syphilis. These prostheses were quite heavy, and by Renner's own admission, very painful to insert. ${ }^{4}$ Lusitanus and Paré expanded on these designs to involve a metal plate and a sponge in the nasal cavity to provide retention - the sponge, once forced into the nasal cavity, expanded. ${ }^{4}$ Paré was the first to use the term obturatuer (in 1575-1585), having first referred to this prosthesis type as a couvercle (or "coverlid'). Very little information was given about their construction. Fauchard, commenting on these obturators, observes 'they fall out and be displaced so easily as to be useless, embarrassing and uncomfortable. ${ }^{10}$ It is also worth noting that both the obturators designed by Lusitanus and Paré could not be easily removed, and that the sponge component quickly became contaminated and malodorous.

Fauchard's text gives clear instruction on the fabrication of five designs of obturators. ${ }^{10}$ The first two designs (Fig. 2) were made for dentate patients, and the

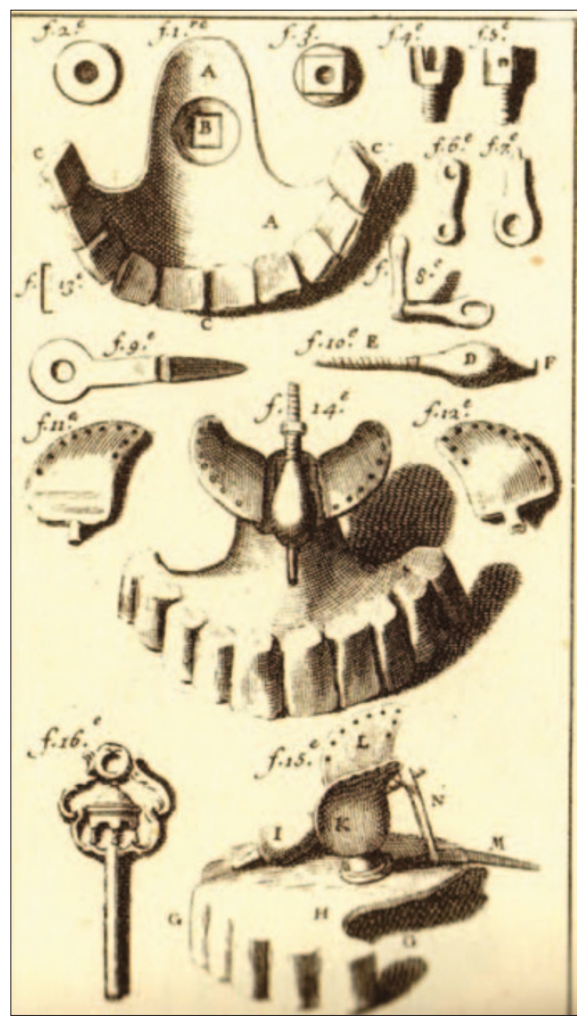

Fig. 3 Plate 39 from Le Chriurgien Dentiste showing the third design, for edentulous patients. The oral component was made from both metal and ivory and included prosthetic teeth, which can be clearly seen. The retentive wings are shown in their horizontal position in the middle of this image.

third (Fig. 3) and fifth (Fig. 4) for edentulous patients. The oral component of the first two designs was metal, while that of the third and fifth was part metal and part ivory, to which prosthetic teeth were attached as required.

Fauchard generally used human, hippopotamus, or ox teeth, or tusks from the walrus or elephant as material from which prosthetic teeth could be made. ${ }^{5}$ The first three and the fifth designs essentially depend on the same retentive principle. Instead of relying on sponges to retain his prostheses, Fauchard used a design consisting of two metal wings that were inserted through the perforation in a vertically upright position. Borrowing an idea from his hobby of watch-making, the metal wings were then adjusted into a horizontal position by means of a threaded screw. These then engaged on the nasal side of the defect, providing retention for the prosthesis.

The fourth design (Fig. 4) was made for a patient who had lost her upper incisor teeth and had a defect that communicated with the floor of the nose. This prosthesis was made of ivory, which was shaped with 'an elevation' that extended into the defect and to which was attached a sponge.

The designs were crude, and it is diffi- 


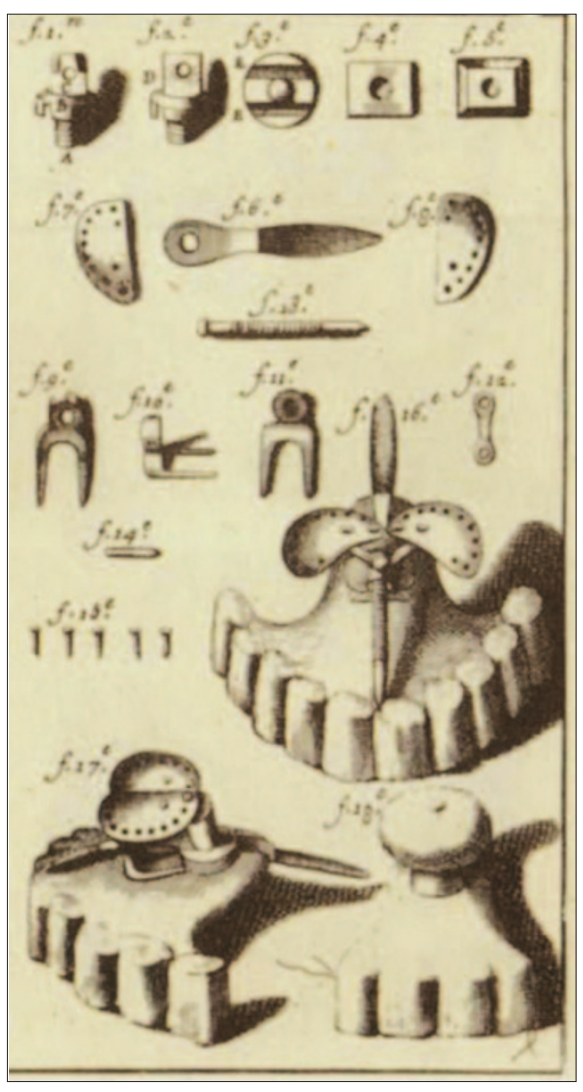

Fig. 4 Plate 40 from Le Chriurgien Dentiste. The fourth design, which was made specifically for a lady who had lost some anterior teeth, is shown at the lower right hand corner of this image. This prosthesis was made of ivory, and included an 'elevation' that extended into the defect and to which was attached a sponge. The fifth design is shown twice, in the middle of this image and in the lower left hand corner. It was made for an edentulous patient. Its oral component was made from metal and ivory. It was again retained by metal wings, and these are shown in a lower position in this image. cult to imagine a modern patient tolerating one of Fauchard's obturators. It is worth remembering that he had not developed impression techniques and could not make models of the defect. Neither did he have access to materials such as silicone, acrylic or gutta percha which are commonly used in the practice of maxillofacial prosthodontics today. But since the alternative was a prosthesis made from leather, gold, or ivory and retained by a sponge that quickly became fetid, then Fauchard's designs must have appeared revolutionary in their day. The appliances would have conferred a significant improvement in the quality of life for his patients compared with the contemporary alternatives. He records his triumph with his obturator designs: '...these are not merely ideas ... they have been brought into practice ... with all the success that I could have hoped... ${ }^{10}$

\section{CONCLUSION}

It is all too easy to dismiss the quality of Pierre Fauchard's dentistry as rudimentary by modern standards. Many of his techniques relied on materials that have been outdated for centuries. But he was the first to publish detailed designs of how to construct an obturator and his designs for obturators were sophisticated and complex. Considering the limitations under which he worked, they were ingenious for their time. Fauchard's overall contribution to modern dentistry was exceptional. He was a creative and innovative thinker, a very caring clinician, and possessed considerable manual skills. He encouraged the sharing of knowledge and ideas among dentists, and wrote the first detailed textbook of dentistry. As a result of Fauchard's efforts, the practice of dentistry was changed immeasurably for the better, and colleagues began to share information with each other.

Without Fauchard's contribution, dentistry would not have evolved so rapidly to attain the status of a leading health care profession. His exemplary and humane approach to patient care was particularly evident in his provision of obturators, a very worthy practice which in those days rehabilitated patients afflicted by trauma or syphilis, and in modern times helps patients suffering from cancer, and those born with congenital defects of the palate.

1. Wiens JP, Wiens R L. Psychological management of the maxillofacial prosthetic patient. In Taylor T D. (Ed) Clinical maxillofacial prosthetics. pp 1-14. Illinois: Quintessence Publishing, 2000.

2. Lynch C D, Allen PF. A modified copy technique fo obturator prostheses. Euro J Prosth Rest Dent 2004; 12: 166-170.

3. Jacob R F. Clinical management of the edentulous maxillectomy patient. In Taylor T D. (Ed) Clinical maxillofacial prosthetics. pp 85-102. Illinois: Quintessence Publishing, 2000

4. Hoffman-Axthelm W. History of dentistry. pp 136-193. Quintessence Publishing, 1981.

5. Viau G. The life of Pierre Fauchard. Dental Cosmos 1923; 65: 797-808.

6. McManus C. Pierre Fauchard. Dental Cosmos 1907; 49: 1233-1245.

7. Viau G. The manuscript of Fauchard. Dental Cosmos 1923; 65: 823-826.

8. Prinz H. Pierre Fauchard and his works. Dental Cosmos 1923; 65: 827-830.

9. Loader C. What is the Pierre Fauchard Academy? Dental Survey 1976; 52: 64-68.

10. Fauchard P. The Surgeon Dentist. Vol. 2. Lindsay L. (Trans) pp 99-113. London: Butterworth \& Co, 1946. 